\title{
[Case Study] SARS-CoV-2 infection in a fully vaccinated case with newly diagnosed breast cancer: a case report
}

\author{
Anna Maria Vandone ${ }^{1}$, Manuela Alessio ${ }^{2}$, Veronica Prati ${ }^{2}$, Giorgia Zucchini ${ }^{2}$, Morena Ciceri $^{2}$, Cinzia Ortega $^{3}$ \\ 1 Azienda Sanitaria Ospedaliera S.Croce e Carle Cuneo \\ 2 Ospedale Alba-Bra \\ 3 Azienda Sanitaria Locale CN2 Alba-Bra
}

Funding: The author(s) received no specific funding for this work.

Potential competing interests: The author(s) declared that no potential competing interests exist.

\section{Abstract}

Background: the novel Severe Acute Respiratory Syndrome Coronavirus (SARS-CoV-2) was identified in 2019 inWuhan, China, and has not been previously identified in humans. SARS-CoV-2 disease resulted in a global pandemic, impacted on public health service and in major changes in the lifestyle and quality of life of population.

Case: we describe a 62-year-old woman with new diagnosis of breast cancer, infected with the SARS-CoV-2, within 14 days of the second dose of the BNT162b2 mRNA COVID-19 vaccine.

Conclusion: in cancer population, timely vaccinations and maintenance of the maximum alert threshold, are necessary to prevent infection spreading.

\section{Introduction}

Coronavirus disease 2019 (COVID-19) has quickly spread worldwide, caused by SARS-CoV-2.

Italy reported its first case of COVID-19 on 29 January $2020^{11]}$ and reached more than 4.5 million cases by October 2021 with more than 130 thousand deaths. In Italy, on October 2021, a total of Pfizer-BioNTech 62.419.804 doses has been administered (Ministry of Health Report).

The Pfizer-BioNTech COVID-19 vaccine was authorized by the European Commission and EMA on 21 December $2020^{[2]}$ and was approved the following day by AIFA (Italian Medicines Agency).

This case report describes a SARS-CoV-2 infection in a Italian female patient, with newly diagnosed breast cancer. Symptomatic infection developed after the second dose of the Pfizer BNT162b2 mRNA COVID-19 vaccine in the beginning of March 2021.

\section{Case Report}


We describe the case of a 62 years old woman with new diagnosis of breast cancer who, on 15 February 2021, received the first dose of the Pfizer BNT162b2 mRNA COVID-19 vaccine and the second one on 8 March 2021. The only side effect after getting COVID-19 vaccine was transitory pain at injection site.

Patient presented good general condition, she suffers for hypertension, treated with olmesartan, and she has a body mass index of 35, she didn't smoke.

On 4 March 2021, between the first and the second vaccine dose, our patient was in close contact with a COVID-19 case. She is a physiotherapist and she regularly wore personal protective equipment as anti-Covid-19 protocol. Contact was close and prolonged in a closed room.

Furthermore, she underwent a core biopsy of right breast on 2 March 2021, with direct contact with clinical staff. The Hospital admission was made with anti-Covid-19 rules and a Stage I right breast cancer was diagnosed.

After become aware of the close contact with a COVID-19 case, our patient underwent COVID-19 rapid antigen swab on 13 March 2021, five day after the second vaccine dose, obtaining a positive result. In that moment, patient had mild symptoms, such as fever $\left(37.5^{\circ}\right)$, asthenia and ageusia. Symptoms were managed at home and resolved completely after 7 days, excluding asthenia which continued for several days.

On 16 March, has been processed the patient's nasopharyngeal swab at the RDI Laboratory for SARS-CoV-2 detection by real-time RT-PCR, obtaining a positive result. On 26 March, she repeated the molecular nasopharyngeal swab, obtaining a negative result. Our patient and his husband (with two negative nasopharyngeal swab) remained in quarantine until after 26 March 2021.

Because of SARS-CoV-2 infection, surgery for breast cancer was first postponed and later performed without sequelae on 14 April 2021 and than she started regular adjuvant treatment.

\section{Discussion}

The report was approved by the local ethical committee of Cuneo (Italy). Informed consent was obtained from the subject involved in the report.

In our report, we describe a mild Covid-19 disease occurred after 5 days since the second dose of the Pfizer COVID-19 vaccine.

Clinical evolution was favourable, with only upper airway symptoms, recover at home and without any radiological assessment or documented hypoxemia.

Patient completed the isolation period at home with her husband who hadn't Covid-19 infection and wasn't vaccinated: the hypothesis is that in these cases a moderate risk of transmission exist and it's proportionate to patient viral copy number.

Breakthrough infection diagnosis, can undermine the trust on vaccination campaigns and preventive measures, but a 
potential risk of infection after vaccination really exist and support the need to continue application of preventive measures.

Variants spreading on national territory could support the appearance of breakthrough infections, despite a normal people immune reaction to vaccination.

With Delta variant spreading, since June 2021 was mandatory the sequencing of samples from patients with Covid-19 infections after a regular vaccination ${ }^{[3]}$.

Phase III trial of Pfizer COVID-19 vaccine ${ }^{4]}$, confirmed a 95\% acquired protection with vaccination regimen with 2 doses administered intramuscularly 21 days apart. This study was conducted principally in a white American population with a median age of 52 years, about $35 \%$ of randomized people were obese. Cumulative incidence of COVID infections starts to diverge since 12 days after first dose, pointing out an early partial immunizing effect. In this study the efficacy between first and second dose was about $52 \%$ and was $91 \%$ within 7 days since the second one. Pfizer COVID-19 vaccine reached full efficacy at least 7 days after dose two.

In literature there are many reports on COVID-19 infections in regularly vaccinated populations in particularly in healthcare workers. A correspondence on May 2021, describes COVID-19 infections at CaliforniaUniversity during vaccination campaign with mRNA vaccines, with a detected infection rate of $0.05 \%$. In this population, $71 \%$ of documented infections occurred within 14 days since first vaccination dose. In this paper, less infections were described after second dose and the majority of that occurred within 7 days. Authors describe an absolute COVID-19 infections risk higher than the risk reported in clinical trials: possible explanations are the application of systematic screening procedures, high infection rate in that period, demographic differences between real-life population and a clinical trial setting and mostly, an high risk of exposure for health workers ${ }^{[5]}$.

Near to efficacy data on mRNA vaccines to prevent SARS-CoV2 infections, there are preliminary data about vaccines ability to attenuate infection and disease in breakthrough events. In a prospective study recently published, in a large cohort of health care and frontline workers, SARS-CoV2 infections in people partially or completely vaccinated, the mean viral RNA load was $40 \%$ lower than in unvaccinated participants. The risk of febrile symptoms was $58 \%$ lower and the duration of illness was shorter ${ }^{[6]}$.

Cancer diagnosis is recognized as an important risk factor for the development of severe COVID-19 disease and infection susceptibility has been linked to cancer localization and extension, with an higher risk in lung cancer population. ${ }^{[7]}$

During pandemic periods, Oncology Departments necessarily introduced changes to deal with emergency with the great risk of induced patient undertreatment with described delays in screening or diagnostic procedures, in treatments administration and follow-up procedures.

The conduction of a rapid and extensive vaccination campaign, should be the starting point to avoid infection resurgence e to ensure the regular continuation of Oncology Departments activities. 
A prospective observational analysis ${ }^{[8]}$ has been conducted on efficacy and safety of Pfizer BNT162b2 mRNA COVID-19 vaccine in a population of cancer patients compared to healthy controls. This study was conducted in London, in a heterogeneous population of 151 cancer patients, 95 of which with solid tumors and $57 \%$ of them at stage IV. Median age for patients with cancer was 73 years and about $35 \%$ of them had gynaecological or breast cancers. Endpoints were to evaluate efficacy and immunogenicity of Pfizer COVID-19 vaccine with the aim to understand if the 21 days boost, is the right choice within an immunocompromised population. The interim analysis demonstrated that in a cancer population, a single vaccine dose is ineffective to induce seroconversion in most patients: at 21 days since first dose, $94 \%$ of healthy controls develops an antibody response while only $38 \%$ of solid cancer patients and $18 \%$ of haematological cancer patients. After 2 weeks since second dose, seroconversion rise to $100 \%, 85 \%$ and $60 \%$ respectively. This study demonstrated that the great difference between healthy and cancer populations, is in the development of a regular immune response rather than the magnitude.

Vaccination in cancer patients is considered a priority: in Italy, a Ministry of Health decree of March 2021, established that cancer patients without remission or during active immunosuppressive treatment or less than six months since therapy interruption, have to be considered as an high frailty population.

With solid malignancies, vaccination timing depend on vaccine availability rather than ongoing treatment (cytotoxic chemotherapy, targeted therapy, immunotherapy or radiotherapy). ${ }^{[9]}$

In patients receiving cytotoxic chemotherapy, the optimal vaccination timing it's not known. With the great variability in therapies and schedules, it's not clear if vaccine can be more effective when administered simultaneously with chemotherapy or in the rest period between cycles, when it's possible to have also white blood cells nadir. The hypothesis is that granulocytopenia doesn't impact significantly on immunological response to vaccine, due to short term of myelotoxicity in patients treated for solid malignancies. Without other scientific evidences, the recommendation is to vaccine when is possible. ${ }^{[9]}$

In our Institution, since 31 March 2021, the vaccination campaign started for every patients candidates to begin therapy or during active treatment for a solid malignancy ${ }^{[10]}$. The Vaccination Center built, permitted a vaccination campaign targeted, continuous and systematic on our patients. In about two months, we obtain a full vaccine coverage of our patients. In addition to the protection of each individual subject, we guaranteed greater protection to all Cancer Center frequenters, were every day, there are numerous therapeutic accesses. The vaccination campaign ended on 14 June 2021, with 470 doses administered: 243 first dose of a mRNA 2-dose vaccine and 227 second doses. The reported difference, is due to the administration of single dose for patients with COVID-19 history at least 3 months before.

\section{Conclusion}

The knowledge about COVID-19 infection, vaccinations and specific treatments are in fast and steady evolution. Covid-19 infections generated serious economic, social and public health problems worldwide, and today we are starting to see these effects. In Oncology Departments great efforts were made to maintain functioning services, to avoid delays and 
therapeutic adaptations, to prevent the possible virus spread in hospitalization and cytotoxic chemotherapy administration areas.

Maximum effort must be implemented to allow high health care standards in cancer population: timely vaccinations, regular continuation of life-saving treatments to improve oncological prognosis despite immunosuppressive effects, continuation of regular diagnostic tests and maintenance of the maximum alert threshold to prevent infection spreading.

\section{References}

1. 'M.R. Capobianchi, M. Rueca, F. Messina, E. Giombini, et al. (2020).Molecular characterization of SARS-CoV-2 from the first case of COVID-19 in Italy. Clinical Microbiology and Infection, vol. 26 (7), 954-956. doi:10.1016/j.cmi.2020.03.025.

2. " "Statement by President von der Leyen on the marketing authorisation of the BioNTech-Pfizer vaccine against COVID-19".. authorisation of the BioNTech-Pfizer vaccine against COVID-19.

3. ^Normativa Sanitaria.

4. 'Fernando P. Polack, Stephen J. Thomas, Nicholas Kitchin, Judith Absalon, et al. (2020).Safety and Efficacy of the BNT162b2 mRNA Covid-19 Vaccine. N Engl J Med, vol. 383 (27), 2603-2615. doi:10.1056/nejmoa2034577.

5. `Jocelyn Keehner, Lucy E. Horton, Michael A. Pfeffer, Christopher A. Longhurst, et al. (2021).SARS-CoV-2 Infection after Vaccination in Health Care Workers in California. N Engl J Med, vol. 384 (18), 1774-1775. doi:10.1056/nejmc2101927.

6. 'Thompson MG, Burgess JL, Naleway AL, et al.. (2021). Prevention and Attenuation of Covid-19 with the BNT162b2 and mRNA-1273 Vaccines. N Engl J Med., vol. 385: 320-329 .

7. 'Garassino MC, Whisenant JG, Huang LC, et al., TERAVOLT investigators. (2020). COVID-19 in patients with thoracic malignancies (TERAVOLT): first results of an international, registry-based, cohort study. Lancet Oncol, vol. 21: $914-$ 922.

8. 'Leticia Monin, Adam G Laing, Miguel Muñoz-Ruiz, Duncan R McKenzie, et al. (2021).Safety and immunogenicity of one versus two doses of the COVID-19 vaccine BNT162b2 for patients with cancer: interim analysis of a prospective observational study. The Lancet Oncology. doi:10.1016/s1470-2045(21)00213-8.

9. a, bSarah DiGiulio. (2021). NCCN Issues Guidance on COVID-19 Vaccination for Patients With Cancer. doi:10.1097/01.cot.0000743568.73608.8d.

10. ^Rete Oncologica Piemontese. (2021). Protocollo Operativo n.2021/0124947 Vaccinazione anti COVID-19 dei pazienti affetti da patologia oncologica. 